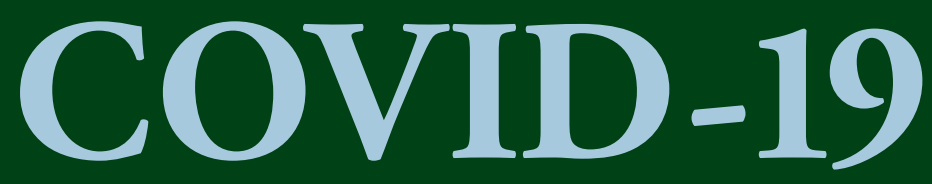

\title{
EINE SOZIAL-
}

\section{WISSENSCHAFTLICHE}

\section{PERSPEKTIVE}

Fiorenza Gamba, Marco Nardone, Toni Ricciardi, Sandro Cattacin (Hrsg.)

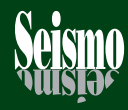

Thomas Abel, Antonio Abellán García, Jean-Michel Bonvin,

Mathilde Bourrier, Claudine Burton-Jeangros,

Sandro Cattacin, Nicola Cianferoni, Ruxandra Oana Ciobanu,

Bernard Debarbieux, Vera de Bel, Maxime Felder,

Fiorenza Gamba, Olga Ganjour, Myriam Girardin,

Marco Nardone, Michel Oris, Loïc Pignolo,

Rogelio Pujol Rodríguez, Diego Ramiro Farinas, Toni Ricciardi,

Emilie Rosenstein, Marlyne Sahakian, Sébastien Salerno,

Daniel Stoecklin, Philippe Wanner, Eric Widmer,

Marie-Eve Zufferey 


\section{COVID-19}

Eine sozialwissenschaftliche

Perspektive

Herausgegeben von

Fiorenza Gamba, Marco Nardone,

Toni Ricciardi und Sandro Cattacin 



\title{
COVID-19
}

\section{Eine sozialwissenschaftliche Perspektive}

\author{
Herausgegeben von \\ Fiorenza Gamba, Marco Nardone, \\ Toni Ricciardi und Sandro Cattacin
}


Veröffentlicht mit Unterstützung der Universität Genf (Abteilung für Soziologie, Institut für soziologische Forschung, Fakultät für Sozialwissenschaften, Unterstützungsfonds für Open-AccessVeröffentlichungen und Allgemeiner Fonds).

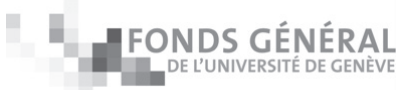

Texte (C) der AutorInnen 2020

(C) 2020, Seismo Verlag, Sozialwissenschaften und Gesellschaftsfragen AG Zürich und Genf buch@seismoverlag.ch www.seismoverlag.ch

ISBN 978-3-03777-219-5 (Print)

ISBN 978-3-03777-748-0 (PDF)

https://doi.org/10.33058/seismo.30748

Das Werk ist lizenziert unter einer Creative Commons

Namensnennung - nicht kommerziell - keine Bearbeitung 4.0 international Lizenz 
«Aber im großen ganzen, fand ich, war diese Seuche solcherart, daß es unmöglich war, die Ansteckung zu entdecken oder ihre Übertragung von einem auf den andern durch irgendeine menschliche Kunst zu verhindern.»

Daniel Defoe, Die Pest zu London (1987 [1722]: 259) 



\section{INHALTSVERZEICHNIS}

ABKÜRZUNGEN

VORWORT

ZUR EINFÜHRUNG: WAS GESCHIEHT UNSERER

GESELLSCHAFT?

Sandro Cattacin, Toni Ricciardi,

Fiorenza Gamba und Marco Nardone

\section{Teil A: Gesellschaftliche DYNAMikeN}

PANDEMIEN IN DER PERSPEKTIVE

DER WELTGESCHICHTE

Toni Ricciardi

Kommunikation EINER PANDEMIE

Sebastian Salerno

KRISE UND KONSUM:

ZURÜCK ZU EINER NACHHALTIGEN ZUKUNFT Marlyne Sabakian

EIN VIRUS TRIFFT AUF DIE

WIRTSCHAFTLICHE DYNAMIK

Jean-Michel Bonvin

DER DEGRADIERTE BETRIEBSMODUS

IN ORGANISATIONEN

Mathilde Bourrier 


\section{TEIL B: ZUGEHÖRIGKEITEN}

OHNE RITUALE KANN MAN NICHT (ÜBER)LEBEN Fiorenza Gamba

SOZIALE DisTANZ UND EINDÄMMUNGSSTRATEGIEN 115 IN COVID-19 ZEITEN

Bernard Debarbieux

DiE ABGESCHOTTETE STADT

Maxime Felder

FAMILIEN UND UNTERSTÜTZUNG AUS DER FERNE Ruxandra Oana Ciobanu

\section{TEIL C: VeRLETZLICHKEITEN}

VERKEHRTE, ZURECHTGERÜCKTE UND

WIEDER HERGESTELLTE STIGMATISIERUNG

Sandro Cattacin

FAMILIENDYNAMIK WÄHREND DES LOCKDOWNS

Eric Widmer, Vera de Bel, Olga Ganjour,

Myriam Girardin und Marie-Eve Zufferey

Die SOZIALE STELlung ÄLTERER MENSCHEN:

OFFENBARUNGEN DURCH DIE KRISE

Michel Oris, Diego Ramiro Farinas,

Rogelio Pujol Rodríguez und Antonio Abellán García

KINDER UND DIE COVID-19-KRISE

205

Daniel Stoecklin

BEHINDERUNG UND DIE ERFAHRUNG VON

VERWUNDBARKEIT DURCH DEN LOCKDOWN

Emilie Rosenstein 


\title{
DIE SOZIALE STELLUNG ÄLTERER MENSCHEN: OFFENBARUNGEN DURCH DIE KRISE
}

\author{
Michel Oris, Diego Ramiro Farinas, \\ Rogelio Pujol Rodríguez und Antonio Abellán García *
}

Seit Beginn der Krise haben sowohl die Medien als auch die Wissenschaft systematisch darüber berichtet: Von der Ansteckung mit dem COVID-19-Virus sind vermutlich alle Erwachsenen betroffen, die schweren Fälle verteilen sich über eine grosse Altersgruppe der zweiten Lebenshälfte, doch die tödlichen Folgen, die Todesfälle, betreffen vor allem ältere Menschen, und zwar in erster Linie diejenigen, die als «hochbetagte Menschen» bezeichnet werden, also Menschen hohen Alters, wie die über Achtzig- und Neunzigjährigen oder gar die über Hundertjährigen. In dieser Hinsicht stiess das neue Virus auf eine Bevölkerungsgruppe, die erst aus dem jüngsten Kapitel der Geschichte des Alterns in reichen Ländern hervorgegangen ist. Die Lebenserwartung, die beispiellose Spitzenwerte erreicht, schien plötzlich durch die Pandemie bedroht zu sein, was den Kern eines Paradoxes trifft: Der Fortschritt der letzten Jahrzehnte veränderte durch die Verringerung der finanziellen Abhängigkeit und die Erhöhung der Gebrechlichkeit die Realität des Alters; gleichzeitig machen soziale Ungleichheiten und interindividuelle Vielfalt die heutigen älteren Menschen heterogener als je zuvor.

Es ist das Aufeinandertreffen zwischen dieser Heterogenität von Lebenssituationen und dem Virus, das Risse in soziale Brüche verwandelt, wobei die Ungerechtigkeit im Angesicht des Todes in der Zeit von COVID-19 dies am grausamsten illustriert. Das Sozial- und Gesundheitssystem scheint hier machtlos zu sein. Die Krise verdeutlicht auch 
den Kontrast zwischen unzureichend vernetzten Komponenten, insbesondere zwischen Spitälern und Pflegeheimen. Letztere stehen im Mittelpunkt der Debatten über die Paradoxien des Ausschlusses von Rentnerinnen und Rentnern, gerechtfertigt durch ihren Schutz, und über die Widersprüche im politischen Diskurs und in den gesellschaftlichen Repräsentationen «des Alters».

Dies sind die Themen, die in diesem (zu) kurzen Kapitel behandelt werden, mit einem Vergleich zwischen der Schweiz und Spanien. Die beiden Länder unterscheiden sich in vielerlei Hinsicht, dabei ist es in schwierigen Zeiten noch leichter, nur zu vergleichen mit dem Ziel, zu bewerten und sich selbst als besser zu betrachten als die anderen. Wir möchten diese Falle vermeiden und diese Übung nutzen, um besser zu verstehen und dadurch auch die Reflexion zu verstärken.

\section{FORTSCHRITT ALS RISIKOFAKTOR: ALTERUNG, LANGLEBIGKEIT UND FRAGILITÄT}

Die Alterung prägt sowohl die Schweiz als auch Spanien. Weit entfernt von Stereotypen, die die COVID-19-Todesstatistiken zu legitimieren schienen, sind sich die beiden Länder in dieser Hinsicht recht ähnlich. Der Anteil der über 65-Jährigen an der Bevölkerung ist gleich hoch (19\%), wenn auch in Spanien der Anteil der über 80-Jährigen $(5,9 \%)$ etwas höher liegt als in der Schweiz $(4,9 \%)$. Viele europäische Staaten haben ähnliche oder höhere Werte, da die demografische Alterung das Ergebnis mehrerer Faktoren und deren Kombinierung ist (Fruchtbarkeit, Sterblichkeit und Migration) während der turbulenten Geschichte des 20. Jahrhunderts. Doch in Bezug auf die Lebenserwartung der Bevölkerung, die mit rund 84 Jahren die zweithöchste der Welt ist, scheint sich die Schweiz abzuheben, denn nur Japan schneidet besser ab. Obwohl diese Position in den Schweizer Medien oft hervorgehoben wird, wird das 
an dritter Stelle stehende Land Spanien (Eurostat-Daten) kaum erwähnt.

In den 1960er Jahren spiegelte die spanische Migration in die Schweiz unter anderem das wirtschaftliche Entwicklungsgefälle zwischen den beiden Ländern wider. Niemand hätte damals vorausgesagt, dass Spanien in Bezug auf die Langlebigkeit seiner Bevölkerung heute so gut abschneiden würde wie die Eidgenossenschaft. In der Schweiz sind sich nur sehr wenige Menschen dieser Tatsache bewusst, während sie in Spanien eine Quelle des Stolzes ist. Im vergangenen Jahr sagten einige sehr seriöse Studien sogar voraus, dass Spanien aufgrund gesünderer Konsumgewohnheiten (weniger Rauchen und Fettleibigkeit, die berühmte mediterrane Ernährung) und der Qualität ihres Gesundheitssystems (Foreman et al. 2018) die Schweiz und Japan überholen und sich weltweit an erster Stelle positionieren würde (Foreman et al. 2018).

Die beeindruckenden erzielten Fortschritte können letztlich auf den Rückgang der Morbidität zurückgeführt werden oder einfacher gesagt: Wir leben länger, weil wir gesünder sind. Tatsächlich liegt die Lebenserwartung bei guter Gesundheit, das heisst, ohne Einschränkung in der Fähigkeit, den Aktivitäten des täglichen Lebens nachzugehen, in Spanien bei 73,80 Jahren und in der Schweiz bei 73,46 Jahren (Werte von 2016). Diese Zahlen sind zwar erfreulich, stehen aber auch für eine düstere Botschaft, da sie etwa zehn Jahre unter der Lebenserwartung liegen, was auf eine entsprechende Phase der Schwierigkeiten oder gar des Leidens hindeutet und sich auch in den negativen Darstellungen des «vierten Alters» wiederspiegelt.

Letzteres wurde lange Zeit als das Alter der Abhängigkeit wahrgenommen, das auf das Alter der Autonomie folgt und somit den Übergang vom sozialen Alter (Ruhestand) zum «wirklichen» (oder biologischen) Alter markiert, oft nach Unfällen, die eine Situation der Behinderung schaffen. Am Ende des 20. Jahrhunderts verfolgte das Team von Christian Lalive d'Epinay (Universität Genf) mehrere Jahre 
lang eine Kohorte von Schweizerinnen und Schweizer über achtzig. Diese Forschenden wiesen auf das beeindruckende Fortschreiten der Fragilität hin, eines Zwischenzustands, der Personen kennzeichnet, die zwar noch selbstständig aber auch Mühe haben und daher oft Hilfe benötigen. Eine Fülle von Studien, die aufgrund der Vielfalt der verwendeten Messgrössen schwer zu vergleichen sind, belegt die Bedeutung einer solchen Lebenserfahrung für den Lebensweg älterer Menschen, was auch im demographischen Ansatz Widerhall findet.

Ein Bild ist mehr wert als eine lange Rede, deshalb verweisen wir den Leser auf Abbildung 1, die die Überlebenskurve nach Alter und Geschlecht in der Schweiz zeigt.

Abbildung 1: Überlebenskurven für Männer und Frauen in der Schweiz im Jahr 2019

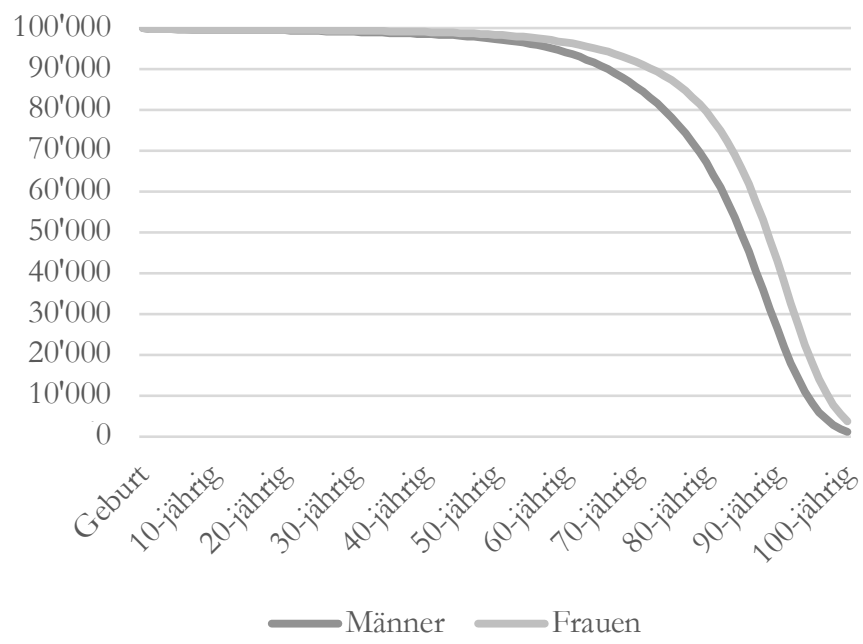

Quelle: Angaben des Bundesamts für Statistik.

Die Abbildung enthält vor allem eine wichtige Information. In unseren fortgeschrittenen Gesellschaften gibt es bis ins 
hohe Alter nur wenige Todesfälle. In der Schweiz sind 2019 drei Viertel der Männer im Alter von 76 Jahren noch am Leben, aber nur ein Viertel davon wird im Alter von 91 Jahren noch am Leben sein; bei den Frauen liegen die Werte bei 82 bzw. 94 Jahren. Die Hälfte aller Todesfälle konzentriert sich daher auf einen Zeitraum von 15 Jahren auf der männlichen Seite und kaum 12 Jahren auf der weiblichen Seite. Aufgrund heutiger Alterungsprozesse gibt es eine fragile Bevölkerungsgruppe, die der Tod innert kürzester Zeit «wegrafft» (Oris und Lerch 2009). Und genau hier schlug COVID-19 zu, wie es in Abbildung 2 für Spanien ersichtlich ist.

Diese Zahlen veranschaulichen, dass Fortschritt auch eine paradoxe Quelle der Ungleichheit ist. Verbesserungen der Lebensbedingungen, der Pflege und der Unterstützung haben somit die Entwicklung einer älteren Bevölkerung ermöglicht, deren bereits zuvor bestehende Zerbrechlichkeit das Virus offenbarte. Die Zahlen deuten auf grosse Mortalitätsunterschiede zwischen Spanien und der Schweiz hin, doch auch nach der Einwohnerzahl gewichtet, hängen die Werte vom Grad des Fortschreitens der Epidemie ab und werden erst nach deren Ende vergleichbar sein. Ausserdem gibt es in beiden Ländern grosse regionale Ungleichheiten, die immer noch wenig untersucht sind.

Letztlich gibt es immer noch so viele Unterschiede bei den Todesursachen, ob sie nun COVID-19 zugeschrieben werden oder nicht, ob nach einem Test oder nicht, dass jeder Vergleich heikel ist und bleiben wird. Das mediane Sterbealter der COVID-19-Patientinnen und -Patienten im Alter von etwa 80/84 Jahren bezieht sich jedoch auf die um 1940 geborenen Generationen, eine für die Spanierinnen und Spanier wesentlich schwierigere Zeit als für die Schweizer Bevölkerung. Lebensverlaufsstudien haben nun gezeigt, dass schlechte Lebensbedingungen in der Kindheit die Wahrscheinlichkeit erhöhen, im Alter viel früher gebrechlich zu werden (Palloni und Beltrán 2015; van der Linden et al. 2019). 


\section{Abbildung 2: Überlebenskurven für Männer und Frauen in}

Spanien. Zahlen für 2018, erhöht um die bis zum 24. April 2020 registrierten COVID-19 Todesfälle

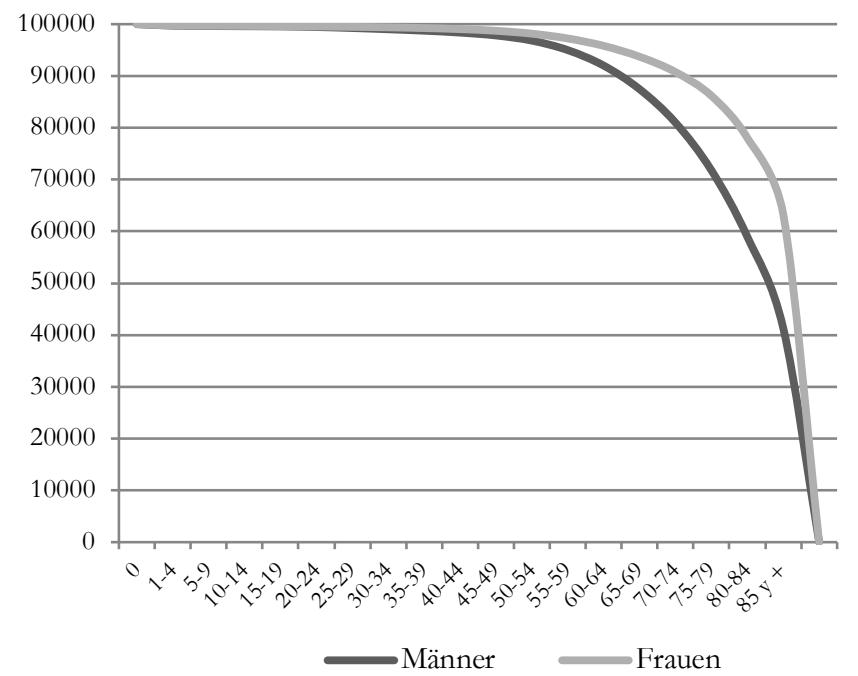

Quelle: Zusammenstellung auf der Grundlage von Bevölkerungszahlen des Nationalen Instituts für Statistik und Daten des Gesundheitsministeriums: Gesundheits- und Notfallwarnzentrum.

Wenn man jedoch in Ländern lebt, die die weltweit höchste Lebenserwartung erreicht haben, bedeutet dies, dass der Tod später zuschlägt. Um ein konkretes Beispiel zu nennen: Ein spanischer Einwanderer, dessen Körper verbraucht ist, nachdem er sein ganzes Leben lang auf Baustellen in der Schweiz gearbeitet hat, hat eine viel grössere Chance als vor 25 oder 30 Jahren den Ruhestand - ja sogar mit 80 oder mehr Jahren das Alter der Gebrechlichkeit zu erreichen.

Noch nie zuvor war die ältere Bevölkerung so heterogen und von sich kumulierenden Ungleichheiten durchzogen (Remund et al. 2019). So ist in der Schweiz, in Spanien und 
auf der ganzen Welt die durch COVID-19 verursachte Sterblichkeit nicht nur mit dem Alter, sondern auch mit Komorbiditäten wie Atemwegs- oder Herz-Kreislauf-Erkrankungen, Diabetes und Bluthochdruck verbunden, wobei die beiden letzteren häufig mit Adipositas in Verbindung gebracht werden (Renova 2020). Im Zentrum der Krise (März 2020) waren rund $80 \%$ der Patientinnen und Patienten auf den Intensivstationen der Genfer und Lausanner Universitätsspitäler adipös.

Adipositas reflektiert sozusagen karikaturhaft soziale Ungleichheiten. Zum jetzigen Zeitpunkt haben wir noch wenig Daten, um die unterschiedliche Sterblichkeit aufgrund von COVID-19 zu messen, aber es scheint belegt, dass das Virus die Ungerechtigkeit des Todes noch akzentuiert hat (siehe auch die Kapitel von Claudine Burton-Jeangros und Philippe Wanner). In Grossbritannien hat sich die bereits in normalen Zeiten höhere Sterblichkeit in den ärmsten Vierteln mehr als verdoppelt. Für die Vereinigten Staaten hat Didier Fassin, Inhaber des Lehrstuhls für öffentliche Gesundheit am Collège de France, am 16. April $2020 \mathrm{im}$ Internet eine Notiz veröffentlicht, in der er auf die überhöhte Sterblichkeit von Afroamerikanerinnen und Afroamerikaner hinweist, die zwei- bis dreimal stärker betroffen sind als der Rest der Bevölkerung. Innerhalb dieser Gruppe summieren sich alle oben genannten Benachteiligungen, insbesondere in einem Land, in dem die Ärmsten in der Regel keine Krankenversicherung haben und die Kosten für Gesundheitsdienstleistungen unerschwinglich sind. In Spanien und der Schweiz ist dies zwar nicht der Fall, doch hat COVID-19 Druck auf die Sozial- und Gesundheitssysteme ausgeübt und deren Mängel aufgedeckt. 


\section{DIE BEHANDLUNG VON UNGLEICHHEITEN: HERAUSFORDERUNGEN FÜR DIE GESUNDHEITS- UND SOZIALSYSTEME}

Angesichts des Ausbruchs der Epidemie reagierten die verschiedenen Staaten mehr oder weniger prompt und beobachteten genau die chinesischen und italienischen Erfahrungen, teils auch mit schuldbewusster Herablassung. Am Ende waren die Massnahmen überall ziemlich ähnlich, mit einem Lockdown, dessen Intensität (hoch in Spanien, moderat in der Schweiz) anscheinend keinen grossen Unterschied auf die Entwicklung der Pandemie ausmachte. Überall war jedoch das Ziel, durch eine Abflachung der Kontaminationskurve eine Überlastung der Notfall- und Intensivstationen zu vermeiden, um nicht unter den Patientinnen und Patienten diejenigen auswählen zu müssen, die nicht behandelt werden würden.

Es ging eindeutig um die Befürchtung, dass ältere Menschen zu Opfern werden könnten, eine Angst, die laut einer kürzlich durchgeführten Umfrage der Hochschule für Soziale Arbeit in Fribourg bei der älteren Bevölkerung in der Westschweiz tatsächlich umging. Die hohe Sterblichkeitsrate von auf Intensivstationen eingelieferten Patientinnen und Patienten ist unbarmherzige Zeugin davon. Trotz unsicherer Prognose haben die Pflegefachpersonen ohne Zweifel ihr Möglichstes gegeben. Dank in Umfang und Geschwindigkeit beeindruckender Reorganisationen, dank dem Einsatz von Freiwilligen, des Zivilschutzes und sogar der Armee, hat sich das Spitalwesen in der Schweiz recht gut gehalten. Im Unterschied dazu wurde dieses in Spanien bis an die äussersten Grenzen ausgereizt, ohne jedoch zu implodieren. All diese in den Rang von Heldinnen und Helden erhobenen Akteurinnen und Akteure verdienen sicherlich mehr als unseren Applaus.

Dieser durch den politischen und medialen Diskurs glorifizierte Einsatz rückte einen ganzen Bereich des facettenreichen Pflegesektors, nämlich denjenigen für sehr alte 
Menschen und die Pflegeheime in den Schatten. Deren Nutzung hängt stark von Familienkulturen ab. In der Schweiz lebten knapp $16 \%$ der Bevölkerung ab 80 Jahren in diesen Pflegeeinrichtungen (Zahlen für 2017), die auf Französisch Etablissements médico-sociaux (EMS) bezeichnet werden, was den medizinischen Aspekt unterstreichen soll.

In den letzten zwei bis drei Jahrzehnten wurde die Pflege zu Hause priorisiert, mit dem Ergebnis, dass zwischen 2012 und 2017 zwar die Bevölkerung im Alter von $80+$ um $22 \%$, die Zahl der Plätze in Alters- und Pflegeheimen jedoch nur um $9 \%$ gestiegen ist. Das durchschnittliche Eintrittsalter hat sich auf 78,8 Jahre für Männer und 82,8 Jahre für Frauen erhöht, in Übereinstimmung mit dem Alter der Gebrechlichkeit und dem damit verknüpften Sterblichkeitsrisiko. Tatsächlich stirbt ein Viertel der Männer innerhalb von drei Monaten nach der Aufnahme, die Hälfte von ihnen innerhalb eines Jahres (gemäss OFS 2019). In Spanien, wo das Familiensystem als «stärken» angesehen wird, betrug der Anteil der über 80-Jährigen in Pflegeheimen nur 8,9\%, ein leichter Anstieg im Vergleich zu 2011.

Im Fall Spanien eskalierte der Skandal um Pflege- und Altersheime im Zusammenhang mit COVID-19 zuerst und rückte deren Strukturen in den Mittelpunkt der Debatte über die Krise und ihr Management. Aufgrund des anfänglich verzerrten Blicks haben viele Staaten Tag für Tag nur Krankenhausstatistiken kommuniziert; andere, wie zum Beispiel Spanien, zählten nur jene Todesfälle, bei denen COVID-19 als Ursache mit einem Test bestätigt wurde. Erst Ende März entsandte die spanische Regierung die Armee zur Desinfektion von Pflege- und Altersheimen. Die Äusserung der Verteidigungsministerin Margarita Robles, dass das Militär «verlassene, wenn nicht sogar tote ältere Menschen in ihren Betten gesehen» habe, rief starke Emotionen sowohl in Familien wie auch in Medien hervor. Die für diese Einrichtungen verantwortlichen Unternehmen wurden (und werden) beschuldigt, Gewinne über das Wohl 
und die Gesundheit ihrer Bewohnenden zu stellen, indem sie und ihre Betreuungspersonen deren Schutz angeblich vernachlässigt haben.

Auch wenn diese Zahlen unsicher bleiben, so wurde doch bis zum 27. April 2020 bei mehr als 5.700 Todesfällen ein positiver COVID-19-Test nachgewiesen; diesen müssten noch weitere 10.800 potentielle Fälle hinzugefügt werden, die zwar Symptome aufwiesen, aber nicht getestet wurden. Wenn man diese Todesfälle auch mit einbeziehen würde, dürfte die von der spanischen Regierung bis zum 24. April gemeldete Zahl von 23.190 Todesfällen im Zusammenhang mit COVID-19 unter Berücksichtigung aller üblichen Vorbehalte eigentlich bei über 34’000 liegen. Die Kontroverse über Todesfälle in Einrichtungen wurde auch in Italien oder in Belgien geführt. Belgien macht das Virus in einer zugegebenermassen sehr umfassenden Statistik für $47 \%$ der Todesfälle in Krankenhäusern und $53 \%$ in $\mathrm{Al}$ tersheimen verantwortlich. So schloss Belgien schliesslich zu Grossbritannien auf, wo das Nationale Statistikamt allein zwischen dem 10. und 24. April die Zahl von 4343 tödlichen Fällen in den landesweiten Einrichtungen auf Verlangen der Presse bekannt gab, nachdem diese die Regierung beschuldigt hatte, die Zahl der Pandemie-Todesopfer absichtlich zu senken.

In einem am 28. April 2020 durch die Tribune de Genève durchgeführten Interview beschreibt Vihn-Kim Nguyen, Professor am Graduate Institute in Genf und ehemaliges Mitglied der Koordinierungs- und Planungsstelle in Quebec, die Situation der Altersheime als apokalyptisch:

«Kranke Menschen werden durch erkrankte Mitarbeitende im Stich gelassen. Wir entdecken dehydrierte Menschen und viele Todesfälle.»

Auf die Bitte, diese Dramen zu erklären, antwortet er ohne jede Schamesröte: 
«Wir schauten weg. Jeder wusste, dass sie sehr verletzlich waren. Dies ist mehr als ein politischer Skandal, denn wir waren alle Komplizen.»

Diese Auslegung beruht auf negativen Darstellungen über das Alter, insbesondere über das hohe Alter und die Senilität, die unsere vom Jugendwahn geprägten Gesellschaften verdrängen, mit dem Ziel, eine uns störende Vision vom Lebensende von uns fernzuhalten. Solche Wahrnehmungen bestehen fort und kommen in Krisenzeiten zum Vorschein. Dennoch sind in den letzten Jahrzehnten echte Anstrengungen unternommen worden. Insbesondere wurde der Wunsch geäussert, das Image der Alters- und Pflegeheime zu verbessern, sie zu in die Quartiere integrierten Lebensräumen zu machen, wo generationsübergreifende Beziehungen gefördert werden und wo Betreuende die Neugierde und die Aktivität der Älteren erhalten.

Zwischen alten, aber beständigen Visionen und neuen Ansätzen können wir die Reaktion eines Verantwortlichen aus dem Kanton Waadt lesen, dem nach dem Tessin am stärksten betroffenen Kanton der Schweiz, in dem die Hälfte der COVID-19-Todesfälle auf Alters- und Pflegeheime zurückzuführen ist. Er räumte ein, dass in der Mehrzahl der Fälle Patientinnen und Patienten aus Pflegeheimen nicht ins Krankenhaus eingewiesen wurden, weil ihre Prognose hinsichtlich Genesung oder Überleben in einem akzeptablen Zustand schlecht oder gar gleich Null war. Es ging also vielmehr darum, für die Begleitung am Lebensende Palliativpflegeteams einzusetzen, als eine Therapie um jeden Preis einzuleiten.

Diese Bemerkungen, die eine gründliche Analyse verdienen würden, wurden eher am Ende des Höhepunktes der Epidemie gemacht, in einer Institution, die nicht über ihre Kapazitäten hinaus gefordert war. In Spanien legten Ambulanzfahrer in einem angespannteren Kontext Zeugnis über weit extremere Situationen ab. In beiden Fällen waren 
es die Spitäler, die Entscheidungen fällten, und die Altersheime, die unter diesen litten. Für letztere hat die Pandemie gar alle jüngsten Bemühungen zunichte gemacht. Vom schlechten Image waren sogar jene Institutionen betroffen, die eigentlich erfolgreich mit der Krise zurechtzukommen mussten, bei denen aber die durch den Lockdown verursachte Isolation und den damit verbundenen Verlust der Lebenslust manchmal «sanfte Selbstmorde» provozierte (gemäss Beobachtungen von Cornelia Hummel im Le Courrier vom 1. Mai); dies wird zweifellos Folgen für die Zukunft haben.

Der Bruch im Diskurs über Ruhestand und Alter ist noch deutlicher geworden, wenn man die zentrale Massnahme des Lockdowns näher betrachtet, die es uns ermöglichte, die Situation wieder in den Griff zu bekommen oder zumindest die Ansteckungen - und damit letztlich auch die Spitaleinweisungen und Todesfälle $-\mathrm{zu}$ reduzieren. Am Ende des zwanzigsten Jahrhunderts entstand das Modell des aktiven Alterns, das seinen Ursprung in der akademischen Welt hatte und dann durch Regierungen und mächtige internationale Organisationen unterstützt wurde. Dazu gehört die Förderung der sozialen Teilhabe und der Beziehungen zwischen den Generationen als Quelle von Gesundheit und individuellem Wohlbefinden: aktives Altern, gesundes Altern!

Doch mit der Einladung, ja sogar Anordnung «zu Hause zu bleiben» und dem Unterbruch des physischen Kontakts zwischen Jugendlichen sowie Erwachsenen und älteren Menschen, die durch die Isolierung zu schützen sind, wurde der Diskurs plötzlich umgekehrt. Diese radikale Umkehrung und diese Einsperrung liegen zwar zweifellos gute Absichten zugrunde, doch sie bringen auch andere $\mathrm{Pa}$ radoxien mit sich.

Auf Anregung von Christian Maggiori führte die Hochschule für Soziale Arbeit in Freiburg die oben bereits kurz erwähnte Umfrage durch. Zwischen dem 17. und 23. April 2020 füllten nicht weniger als 2480 Personen im Alter von 
$65+$ einen Online-Fragebogen aus. Es liegt an den Autoren, die reichhaltigen und nuancenreichen Ergebnisse im Detail zu beschreiben. Was uns jedoch hier direkt betrifft, ist der in dieser Bevölkerungsgruppe vorherrschende Eindruck, dass in den Medien ältere Menschen als für die Krise verantwortlich gemacht wurden, weil wegen ihrem Schutz das soziale und wirtschaftliche Leben der gesamten Bevölkerung gestoppt wurde.

Eine Mehrheit fühlte sich seit Beginn der Krise anders behandelt und ein Fünftel spürte sich gegenüber eine negative Haltung Die Debatte, die anfangs eher implizit geführt wurde, gewann im April 2020 zunehmend an Transparenz, als es darum ging, das Ende des Lockdowns zu konzipieren. Könnten differenzierte Massnahmen ergriffen werden oder mit anderen Worten, könnten bestimmte Gruppen davon ausgeschlossen werden, um sie zu schützen? Schliesslich wurde die Heterogenität der Bevölkerung ab 65 Jahren deutlich. In einer Zeit, in der man erst später vermutete, dass Kinder nicht vom Virus betroffen seien, verdeutlichen deren teilweise auch mystifizierten und verherrlichten Beziehungen zu ihren Grosseltern, nicht nur den gesellschaftlichen Beitrag älterer Menschen und deren soziale Einbettung, sondern auch die emotionale Dimension sozialer Beziehungen (siehe Daniel Stoecklins Kapitel).

\section{SCHLUSSFOLGERUNG}

Jede Krise führt auch zu einem Entwicklungsschub, die aktuelle Pandemie bildet da keine Ausnahme. COVID-19 hat die nach wie vor tiefgreifenden Ambivalenzen hinsichtlich sozialer Darstellungen des Alters und älterer Menschen erneut zum Vorschein gebracht. Über das grosse Engagement des Gesundheitspersonals hinaus, hat die Heroisierung der Spitäler unserer Länder, die Tempel der modernen Technologie, zweifellos auch zu einer impliziten Bitte an 
die Wissenschaft geführt, nämlich das Leben von Menschen zu retten. Die Situation in den Alters- und Pflegeheimen wurde dabei in den Schatten gestellt, oft gar bis es zu spät war. Im Grossen und Ganzen schienen die verschiedenen Teile des Sozial- und Gesundheitssystems unzureichend koordiniert. Es ist jetzt von entscheidender Bedeutung, zu berücksichtigen - vor allem in den Ländern, die die höchste Lebenserwartung der Welt hatten und auch wieder erreichen werden -, was die jüngsten Dynamiken der Alterung in Bezug auf Fragilität und die Vielfalt individueller Situationen, die sich nicht auf ein chronologisches Alter reduzieren lassen, letztlich implizieren. Allgemeiner gesagt: Es wird an uns liegen, nach dieser Pandemie, einen erschütterten Generationenvertrag neu zu schreiben.

* Diese Forschung wurde unterstïtrt durch Longpop (Methodologie und Data-Mining-Techniken für die Analyse grosser Datenmengen auf der Grundlage demographischer und epidemiologischer Längsschnittregister), ein Projekt, das vom Forschungs- und Innovationsprogramm H2O2O der Europäischen Union durcb den Marie Sklodonska-Curie-Fonds 676060 finanziert wurde, und durch das Projekt Überleben und Gesundheit während dem Lebensayyklus RTI2018-097812-B-I00, das vom Ministerium für Wi issenschaff, Innovation und Universitäten, Spanien, finanziert wurde.

\section{LITERATUR}

Foreman Kyle J., Neal Marquez, Andrew Dolgert et al. (2018). "Forecasting life expectancy, years of life lost, and all-cause and cause-specific mortality for 250 causes of death: reference and alternative scenarios for 2016-40 for 195 countries and territories." The Lancet 392(18) : 2052-2090.

OFS, Office fédéral de Statistique (2019). Population des établissements médico-sociaux en 2017. Neuchâtel: OFS.

Oris, Michel und Matthias Lerch (2009). "La transition ultime. Longévité et mortalité aux grands âges dans le bassin lémanique", in Oris, Michel et al. (Hg.). Transitions dans le parcours 
de vie et construction des inégalités. Lausanne: Presses Polytechniques et Universitaires Romandes, S. 407-432.

Palloni, Alberto und Hiram Beltrán-Sánchez (2015).

"Demographic consequences of Barker frailty", in Schoen, Robert (Hg.). Dynamic Demographic Analysis. Cham: Springer, S. 3-20.

Remund, Adrien, Stéphane Cullati, Stefan Sieber, Claudine Burton-Jeangros, Michel Oris und Swiss National Cohort (2019). "Longer and healthier lives for all? Successes and failures of a universal consumer-driven health care system." International Journal of Public Health 64(8): 1173-1181.

RENOVA, Red National de Vigilancia Epidemiológica (2020). Informe sobre la situación de COVID-19 en España. Informe COVID-19, 26, 27 de abril de 2020.

Van der Linden, Bernadette W. A., Stefan Sieber, Boris Cheval et al. (2019). "Life-course circumstances and frailty in old age within different European welfare regimes: a longitudinal study with SHARE”. J Gerontol B Psychol Sci Soc Sci XX(XX): 1-10. 